TITLE:

\title{
Kinetic Interpretation of Rheological Behavior of High Polymers
}

$\operatorname{AUTHOR}(\mathrm{S})$ :

Furukawa, Junji

\section{CITATION:}

Furukawa, Junji. Kinetic Interpretation of Rheological Behavior of High Polymers. Bulletin of the Institute for Chemical Research, Kyoto University 1961, 39(1): 20-34

ISSUE DATE:

1961-03-10

URL:

http://hdl.handle.net/2433/75787

RIGHT: 


\title{
Kinetic Interpretation of Rheological Behavior of High Polymers
}

\author{
Junji Furukawa* \\ (Oda Laboratory) \\ Received September 29, 1960
}

\begin{abstract}
Rheological behavior of high polymers is interpreted in terms of the pseudo cross-linking hypothesis, i.e. polar groups in polymer forms a kind of pseudo-links and contributes to the elastic property of polymer, but such a crossbond as this is not so firm that plastic flow of polymer may be accompanied in some extent. The ratio of real elongation of polymer chain to overall elongation of specimen is demonstrated as the function of slippage of polymer chain.

Creep and stress relaxation are accounted for as the phenomena involving slippage of chdin accompanied by the elongation of chain and the dissociation of cross-bonds. The stressstrain relation in the ease of constant rate drawing are supposed to show the existance of maximum stress according to the hypothesis.

Rupture of linear high polymer are also discussed supposing the breakdown of the second cross-bond and the breakdown may be concluded when load applied is larger than the maximum value estimated from the equation for the equilibrium cross-linking. The rate of rupture is also discussed and the logarithmic life time in rupture is concluded to be correlated linearly to the load applied.
\end{abstract}

\section{INTRODUCTION}

A number of approaches to the rheological behavior of high polymers have been done from several points of view, such as statistical theory as well as mechanical one. Kinetic or rate process theory established by Eyring-Tobolsky school ${ }^{1}$ ) is also one of them and this theory seems to be often convenient to account for the complicated phenomena. The author has proposed some years ago a theory ${ }^{2}$ ) based on the rate process by introducing pseudo-network structure into linear high polymer.

\section{PSEUDO CROSS-LINKED STRUCTURE OF POLYMERS}

This hypothesis is based on the assumption that resinous material is reinforced markedly by the intermolecular forces due to polar groups on polymer chain and this interaction effect may be regarded as a kind of cross-linking effect, although the cross-linking is a temporary one and not chemical cross-linking in vulcanized rubber. Simple treatment of such pseudo or temporary link becomes possible by applying the rate process theory similar to that used in gaseous reaction. Taking $N_{0}$ and $\nu$ as the total number of segment and cross-linked one in unit volume of specimen respectively, the change of cross-linking is generally expressed by equation (1),

$$
d \nu / d t=k_{0}\left(\mathrm{~N}_{0}-\nu\right)-k_{0}{ }^{\prime} \nu,
$$

where $k_{0}$ and $k_{0}{ }^{\prime}$ are rate constants for formation and disappearance of cross-linking

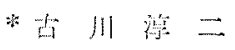


respectively and the former rate is simply assumed to be proportional to remaining uncross-linked segment $\left(\mathrm{N}_{0}-\nu\right)$ and the latter rate is proportional to the links already formed.

The equilibrium concentration of links is expressed by equation (2):

$$
\nu / \mathrm{N}_{0}=1 /\left(1+k_{0}{ }^{\prime} / k\right)
$$

and depends on the temperature as is shown in equation (3):

$$
\nu / \mathrm{N}_{0}=1 /\left(1+\exp ^{-T^{\prime} / R^{T}}\right) .
$$

Here, $\Delta F$ is energy for breaking of pseudo bond, which is defined according to the following equation concerning equilibrium constant $K$ :

$$
K=k_{0}{ }^{\prime} / k_{0}=\mathrm{e}^{-F / R x^{\prime}}
$$

Equation (3) accounts well for the properties at low temperature, since pseudo crosslinking increases exceedingly at low temperature and the material becomes much harder.

\section{Plasticizer EFFeCT}

The purpose of plasticizer is lowering of visco-elasticity of polymers and by using our hypothesis, its action may be ascribed to the decreasing effect on the pseudo-cross-link. If the plasticizer acts merely as a diluent, the corresponding crossbond tends to decrease proportionally to its volume fraction $X$ as is afforded by equations (5) and (6):

$$
\begin{aligned}
& \nu / \mathrm{N}_{0}=\left\{1 /\left(1+k^{\prime}\right)\right\}(1-X) \\
& \nu / \mathrm{N}_{0}=\left(\nu / \mathrm{N}_{0}\right)_{0}(1-X) .
\end{aligned}
$$

Here, $\left(\nu / N_{0}\right)_{0}$ refers to the cross-bond of pure polymer. However, actually the efficiency of plasticizer is not always the same and seems to be larger rather than that calculated from equation (5), and consequently an another additional effect must be taken into account. If the plasticizer of large solvation ability to polymer is used, it may be to prevent the formation of cross-linking in polymer and the following equation may be taken for this instead of equation (1):

$$
d \nu / d t=k_{0}\left\{\mathrm{~N}_{0}(1-X)-\nu\right\}-k_{0}{ }^{\prime}(1-X) \nu-k_{0}{ }^{\prime \prime} X_{\nu} .
$$

In equation (7) the total number of actual segment is taken as $\mathrm{N}_{0}(1-X)$ by considering the diluent effect of plasticizer and the breaking of cross-link is assumed to be attributed to the attack of unlinked segment on linked one as well as that of plasticizer on linked one, where the rate constant for the latter is denoted by $k_{0}{ }^{\prime \prime}$. These effects may be described by the second and the third term in the right side of equation (7). The equilibrium number of linked segments is thus derived as follows:

$$
\nu / \mathrm{N}_{0}=k_{0} \mathrm{~N}_{0}(1-X) /\left\{k_{0}+k_{0}{ }^{\prime}(1-X)+k_{0}^{\prime \prime} X\right\}
$$

If the equilibrium constants for both types of link breaking are

$$
\begin{aligned}
& k_{0}{ }^{\prime} / k_{0}=K^{\prime} \\
& k_{0}{ }^{\prime \prime} / k_{0}=K^{\prime \prime}
\end{aligned}
$$

respectively, equation (8) is transformed into the simple equation (9): 


$$
\begin{aligned}
& \frac{\nu^{\prime}}{\mathrm{N}_{0}}=\frac{1-X}{\left\{1-X\left(K^{\prime}-K^{\prime \prime}\right) /\left(1+K^{\prime}\right)\right\}\left(1+K^{\prime}\right)} \\
& \mathrm{N}^{\nu} \cong\left(\begin{array}{c}
1 \\
1+K^{\prime}
\end{array}\right) \mathrm{e}^{-\frac{K^{\prime \prime}+1}{K^{\prime}+1} X}
\end{aligned}
$$

In the case of $X=0$, equation (9) is, of course, the same as equation (2) and if $K^{\prime}$ $=K^{\prime \prime}$, it is rewritten similarly as equation (5). The latter equation means that the plasticizer having no special solvation effect acts merely as diluent. From equation (9), the linear relationship is suggested between the logarithm of elasticity $E$ and the volume fraction of plasticizer, and the experiment proved to agree with this prediction as shown in Fig. 1.

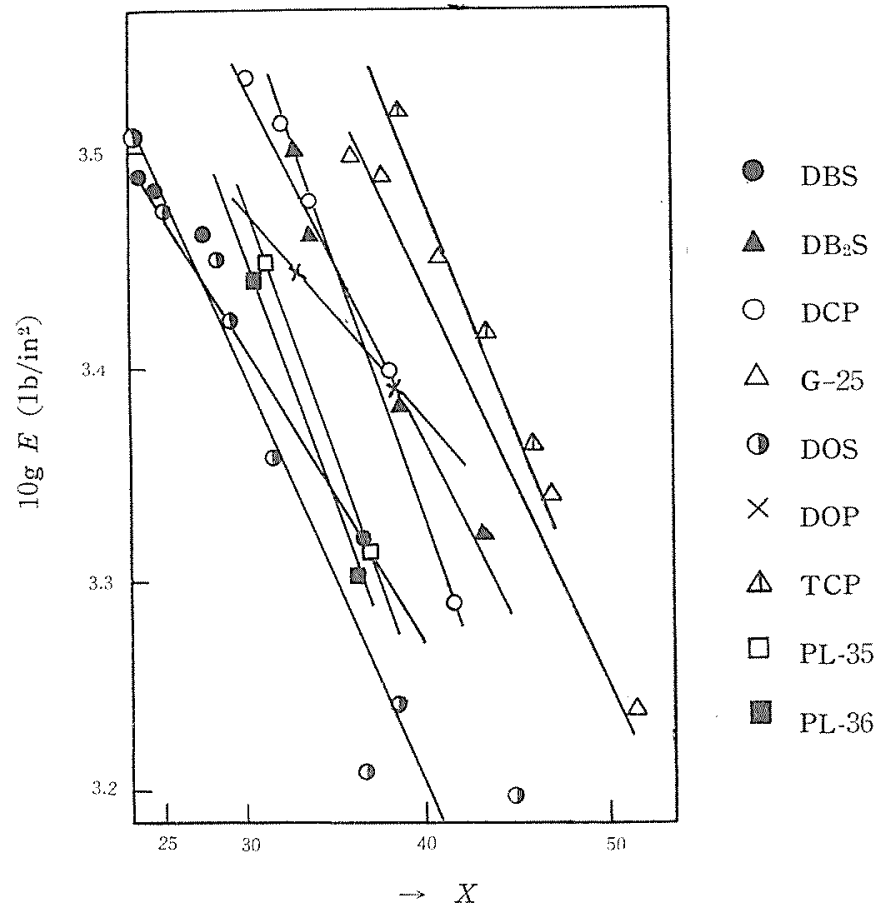

Fig. 1. Linear relationship between logarithmic stiffness of polyvinyl chloride and the added amount of various plasticizers (from Rider's data ${ }^{12}$ ).

In this figure the solvation tendency will be known from the gradient of straight line, which corresponds to $-\left(K^{\prime \prime}+1\right) /\left(K^{\prime}+1\right)$.

\section{EXTERNAL DEFORMATION AND INTERNAL ONE ${ }^{3)}$}

According to pseudo-link hypothesis mechanical behavior of polymeric material can be interpreted as the slippage of cross-links and chain elongation. In creep process the elongation of internal chain is smaller than the external elongation of specimen unlike in the case of vulcanized rubber. Cross sectional area of specimen decreases both in plastic flow and in elastic deformation. If visco-elastic flow is 
analyzed into two processes consisting of plastic and elastic one and cross sectional area is decreased from $A_{0}$ of original state to $A$ after plastic flow, and to $A^{\prime}$ by chain elongation, the length of specimen $\alpha$ and that of chain distance $\lambda$ are expressed by equations (10) and (11) respectively, if the volume remains unchanged during each processes,

$$
\begin{aligned}
& A_{0} / A^{\prime}=\alpha \\
& A / A^{\prime}=\lambda
\end{aligned}
$$

and

$$
\lambda / \alpha=A / A_{0}
$$

Fig. 2 illustrates these relations. If plastic flow is assumed to be expressed by the slippage of pseudo-link and

$$
d A / d t=-k_{0}{ }^{\prime} A,
$$

it followes that

$$
\ln \left(\lambda / \lambda_{0}\right)=\ln \left(\alpha / \alpha_{0}\right)-k_{0}{ }^{\prime} t
$$

or

$$
d \lambda / d t=(\lambda / \alpha)(d \alpha / d t)-k_{0}{ }^{\prime},
$$

where $k_{0}{ }^{\prime}$ is the rate constant of slippage of links.
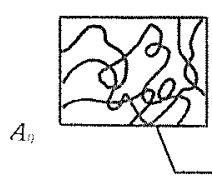

A

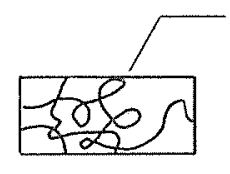

Fig. 2. Model of visco-elasticity on elastomer.

Equation (15) shows the relation between external deformation and internal one, but it should be applied only in the case where the load applied is sufficiently small, because the rate of slippage $k_{0}{ }^{\prime}$ is not constant but it increases by load. According to Eyring's rate theory ${ }^{4}$ ) slippage under large load is to be written as

$$
K_{\mathrm{f}}^{\prime}=k_{0}{ }^{\prime} \exp \left(-\frac{\mathrm{f}}{A} \cdot \frac{\mathrm{a}^{*} / \mathrm{a}}{2 \nu k^{\mathrm{T}}}\right)
$$

where $K_{r}^{\prime}$ is the rate constant under load $f$, and $a^{*}$ and $a$ denote the segment length at transition and ordinary state respectively (see Appendix I).

\section{STRESS RELAXATION UNDER CONSTANT STRAIN}

According to the theory of elasticity of rubber the stress $f$ under elongation is given as:

$$
f=\nu k T A\left(\lambda-1 / \lambda^{2}\right) \text {. }
$$

Consequently stress relaxation can be derived from equations (14) and (17), if $\lambda$ is taken to be constant. 
$\ln f / f_{0}=-2 \mathrm{k}_{0}{ }^{\prime} t$

when $f_{0}$ is the initial stress. Equation (18) corresponds to the equation for Maxwell model.

In the case of large stress, however, equation (16) should be applied and the result will be more complicated, as shown in equation (19):

$$
\ln \left(f / f_{0}\right)=-2 k_{0}{ }^{\prime} \exp \left(f a^{*} / 2 \nu k T A a\right) t
$$

or

$$
\ln \left[\ln \left(f_{0} / f\right)\right]=\ln \left(2 k_{0}{ }^{\prime}\right)+\ln t+f a / 2 \nu k T A a .
$$

If $f$ is so large enough to neglect the term in the left side of equation (20), it is simplified as follows:

$$
f a^{*} / 2 \nu k T A a=\text { const }- \text { In } t \text {. }
$$

Equation (21) has a similar form to that of the equation proposed previously by Eyring-Tobolsky ${ }^{13}$, in which the linear relationship between $f$ and $\ln t$ was pointed out.

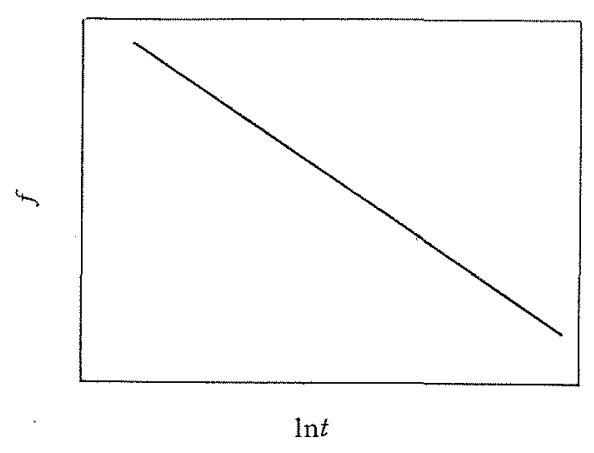

Fig. 3. Relationship between large stress, $f$ and logarithmic time, $\ln t^{5}$.

\section{CREEP UNDER CONSTANT LOAD}

Deformation under constant load can be expressed by equation (14) or equation (22):

$$
\alpha=\left(f_{0} / \nu k T A_{0}\right) \exp \left(k_{0}^{\prime} t\right)
$$

or

$$
\alpha=\alpha_{0} \exp \left(k_{0}{ }^{\prime} t\right) \text {. }
$$

Equation (22) can be derived from equations (13) and (17), and it expresses the increasing rate of deformation with increasing time. Actual creep is however known to have an equilibrium nature, which is possibly expressed by equation (23) instead of equation (15)

$$
\frac{d \lambda}{d t}=\left(\frac{\lambda}{\alpha}\right)\left(\frac{d \alpha}{d t}\right)-2 k_{0}{ }^{\prime}(\lambda-1)+2 k_{0}
$$

where $k_{0}$ is the rate constant of bond fomation (see Appendix II). In the 
latter case the equation for creep is more complicated than equation (13), as is shown in Fig. 4.

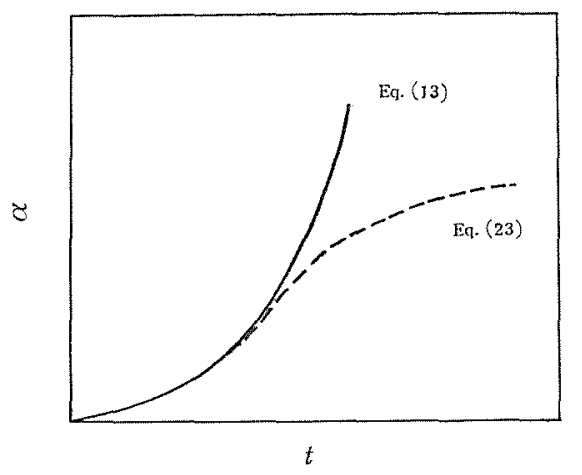

Fig. 4. The comparison between the Equations (13) and (23).

\section{STRESS INTRODUCED BY DEFORMATION OF CONSTANT RATE}

In deformation $\alpha$ of constant rate $\ddot{\alpha}, \alpha$ given by equation (24):

$$
\alpha=\dot{\alpha} t+1
$$

Since stress introduced cross-sectional area $A$ are represented by equations (25) and (26) respectively,

$$
\begin{gathered}
f=\nu k T A \lambda=\nu k T A_{0} \alpha\left(A / A_{0}\right)^{2} \\
A / A_{0}=\mathrm{e}^{-k_{0}{ }^{\prime} t},
\end{gathered}
$$

the dependence of deformation rate on stress is derived as follows:

$$
\ln \left(f / f_{0}\right)=\ln \left(\alpha-1 / \alpha^{2}\right)-2 k_{0}{ }^{\prime}(\alpha-1) / \dot{\alpha} .
$$

It is very important that stress reaches to maximum, $i . e$.

$$
\ln \left(f / f_{0}\right)=\ln (\dot{\alpha} \tau / 2)-2 k_{0}{ }^{\prime}(\tau / 2-1 / \dot{\alpha})
$$

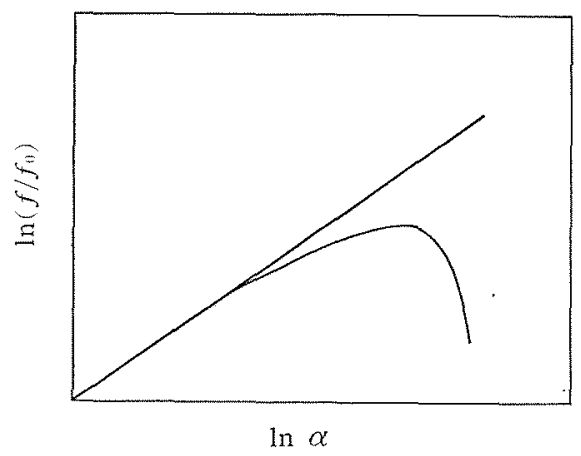

Fig. 5. The relationship between $\ln \left(f / f_{0}\right)$ and $\ln \alpha$.

at such an elongation or time described in equation (28) or equation (29):

$$
\alpha=\dot{\alpha} \tau / 2
$$

or 


$$
t=\tau / 2-1 / \dot{\alpha} \text {. }
$$

Some experiments in literature showed the existence of maximum stress as shown in Fig. 6.

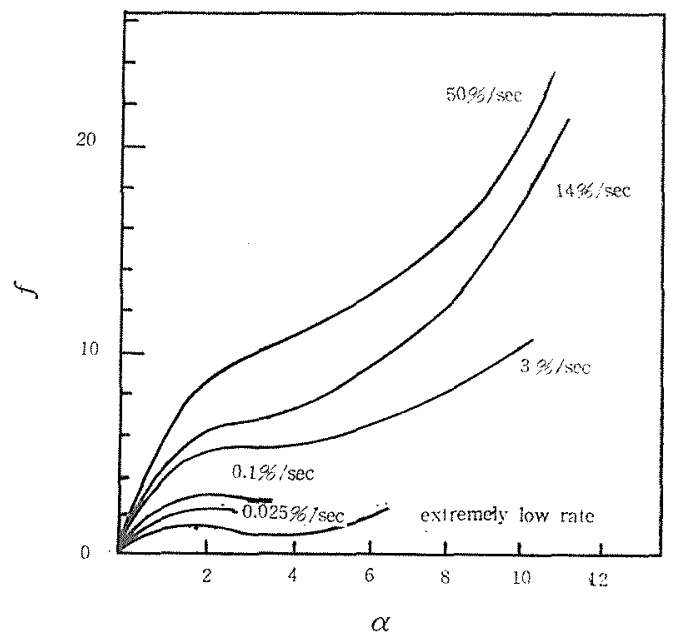

Fig. 6. Stress-strain curve of raw rubber drawn at constant rate (from the data of T.Sakai ${ }^{(3)}$.

\section{RUPTURES OF HIGH POLYMERIC SUBSTANCES ${ }^{3,73}$}

Before discussion it might be noted that polymeric substances undergo rupture by the two different mode, one is the rupture in plastic flow and the other is that in elastic deformation. For an example the plastic substance like chewing gum exhibits the plastic rupture when it is drawn slowly, while in rapid drawing the brittle rupture takes place. In the former case cross section after rupture has a smooth, round or sharp shape, while in the latter case the grossy fractured one, as is illustrated in Fig. 7.

(A)
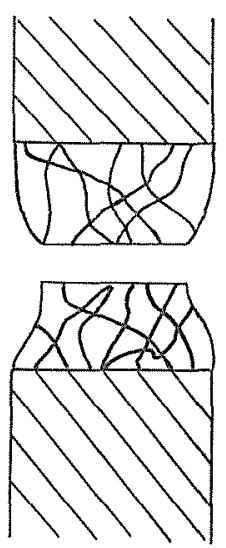

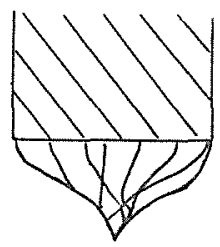

(B)

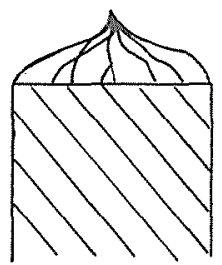

Fig. 7. Brittle rupture (A) and plastic one (B). 
The author assumed that the plastic rupture is attributed to the breakdown of the secondary bond in pseudo-cross-linking and the brittle one to the breaking of the primary bond in the main chain of polymer.

The following is an interpretation for the plastic rupture in terms of pseudocross-linked hypothesis. As mentioned before the equlibrium number of links $\nu$ is expressed by equation (3):

$$
\nu / \mathrm{N}_{0}=1 /\left(1+\exp ^{-E / R T^{T}}\right)
$$

Equation (3) is valid in the case of no stress on the specimen and in the case of rupture under large stress it may have marked influence upon the slippage of the pseudo links, and equilibrium links $\nu_{f}$ can be derived as follows:

$$
\nu_{f} / \mathrm{N}_{0}=1 /\left[1+\exp \left\{-\frac{\Delta F ! \mathrm{N}_{0}(f / A)\left(a^{*} a\right)}{R T}\right\}\right] .
$$

Equation (30) involves the stress effect on dissociation energy of links as designated by the term $\mathrm{N}_{0}(f / A)\left(a^{*} / a\right)$, which means the work applied to one links during slipping of bond, and this equation suggests the existence of maximum value $f_{c}$ for stress at the critical point $\nu_{c}$, as illustrated in Fig. 8.

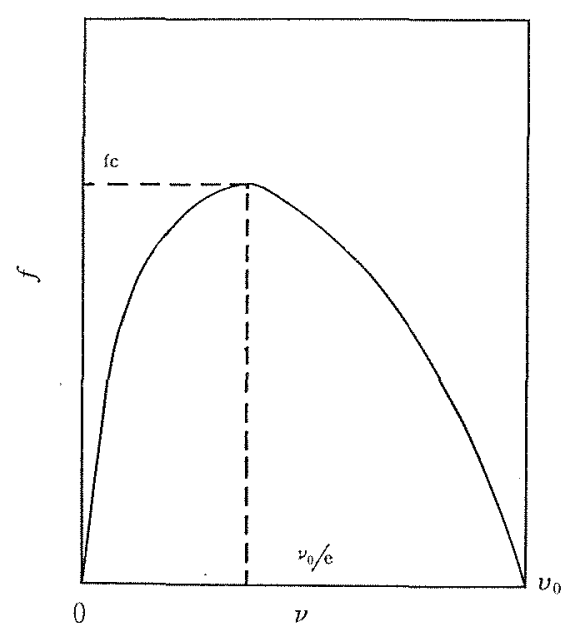

Fig. 8. The relationship between $f$ and $\nu$.

Calculating both $f_{0}$ and $\nu_{c}$ from equation (30), it follows that:

$$
\mathrm{N}_{0}-\nu_{\mathrm{c}}=\ln \left(\frac{\mathrm{N}_{0}-\nu_{\mathrm{c}}}{\mathrm{N}_{0}}\right)+\frac{\Delta F^{\prime}}{R T} .
$$

By inserting equation (3) into equation (31), equation (32) is obtained,

$$
\ln \left(\frac{N_{0}}{\nu_{\mathrm{e}}}-1\right)=\ln \left(\frac{N_{0}}{\nu_{0}}-1\right)+\frac{1}{1-\left(\nu_{\mathrm{e}} / \mathrm{N}_{\mathrm{c}}\right)} \text {. }
$$

Since $\nu_{c} / \mathrm{N}_{0}<1$, equation (32) is simplified as follows:

$$
\ln \left(N_{0} / \nu_{\mathrm{c}}\right) \cong \ln \left(N_{0} / \nu_{0}\right)+1
$$

or

$$
\nu_{\mathrm{c}} \cong \nu_{0} / \mathrm{e}
$$


Equation (34) means that the increase of force decreases the number of links, however it reaches maximum value when the link decreases to the e-th of the original one. If the larger force is applied beyond this critical value, equilibrium link exists no longer. In other words the disappearance of links does not stop and causes the rupture. If the load is smaller than the critical value, plastic slippage of links takes place smoothly according to its equilibrium. The critical force $f_{c}$, which introduces rupture, is given by equation (35):

$$
\mathrm{f}_{\mathrm{e}} / A=\left(a^{*} / a\right)\left(\nu / \mathrm{N}_{0}\right)(1 / \mathrm{e}) R T \text {. }
$$

It is concluded that strength of specimen is to be proportional to the initial number of links, because $\nu_{0}$ is a function of temperature, and strength depends, of course, on temperature, too. If a larger stress than the critical value is applied, the slippage continues infinitely and causes to rupture. The time required for the rupture is known to decrease with increasing load, and it can be predicted by the following consideration. The life time $t_{b}$, which is defined as the time required for rupture, is simply given by the equation (36):

$$
t_{1}=\nu_{0} /-(d \nu / d t) \text {. }
$$

Here the rate of disappearance of links is expressed by equation (16), and it follows that:

$$
t_{\mathrm{b}}=\left(\nu_{0} / k_{0}{ }^{\prime}\right) \exp \left\{-\frac{f}{A} \quad \frac{a^{*} / a}{2 \nu k T}\right\}
$$

$A$ and $\nu$ are not always constant during flow process, but equation (37) suggests the roughly linear relationship between $\log t_{1}$ and $f$. This conclusion is in good accordance with the experimental results, as shown in Fig.9.

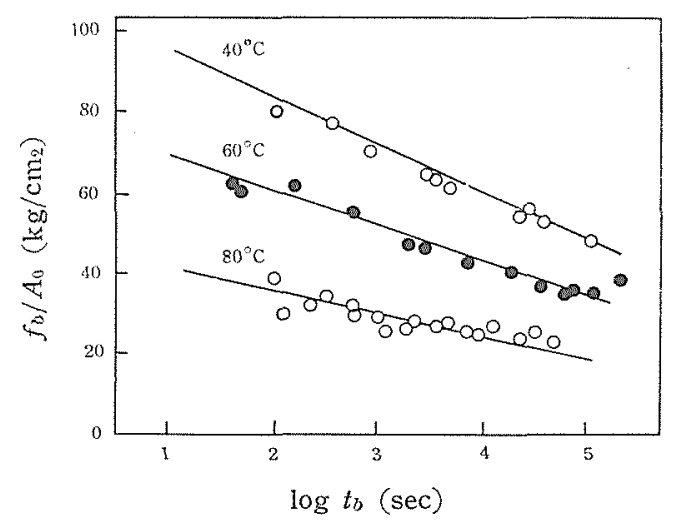

Fig. 9. The linear relationships between strength and logarithmic life time in polyvinylchloride plasticized with dioctylphthalate (100:80) at various temperatures (experiment in our laboratory $y^{8)}$ ).

\section{ELASTIC RUPTURE ${ }^{9}$}

Elastic rupture is attributed to the breaking of primary chemical bond in vulcanized rubber and in this case the strength $f_{b}$ is regarded to be proportional to cross-sectional area $A^{\prime}$ after elongation as well as the number of chain $\nu^{\prime}$ in unit 
cross-sectional area, i.e.:

$$
f, c A^{\prime} y^{\prime} \text {. }
$$

Since chemical cross-linking cannot slip in deformation process, total number of chain in cross section remains unchanged and is equal to the initial values, $A_{0} \times \nu_{0}^{\prime}$.

$$
A^{\prime} y^{\prime}=A_{0} \nu_{0}^{\prime}
$$

Accordingly equation (38) becomes to equation (39)

$$
f_{\mathrm{b}} / A_{0}=f_{0} \nu_{0}^{\prime} \text {, }
$$

where $f_{0}$ is defined as the strength of primary bond. The statistical treatment results the following relation (see Appendix II),

$$
\nu_{0}^{\prime}=\nu_{0} a \text {. }
$$

Taking $a^{*}$ is bond expansion from its original length, equation (31) becomes as follows:

$$
f_{\mathrm{b}} / A_{0}=\nu_{0} a^{*} f_{\mathrm{b}}=\left(a / a^{*}\right) \nu_{0} F_{0} / \mathrm{N}_{0}
$$

Here $\mathrm{a}$ is bond length and $F_{0}$ is dissociation energy of primary bonds. From equation (41) it can be seen that strength is proportional to the bond dissociation energy itself at constant degree of vulcanization, and this conclusion was proved in our experiments on vulcanized rubber as shown in Fig.10.

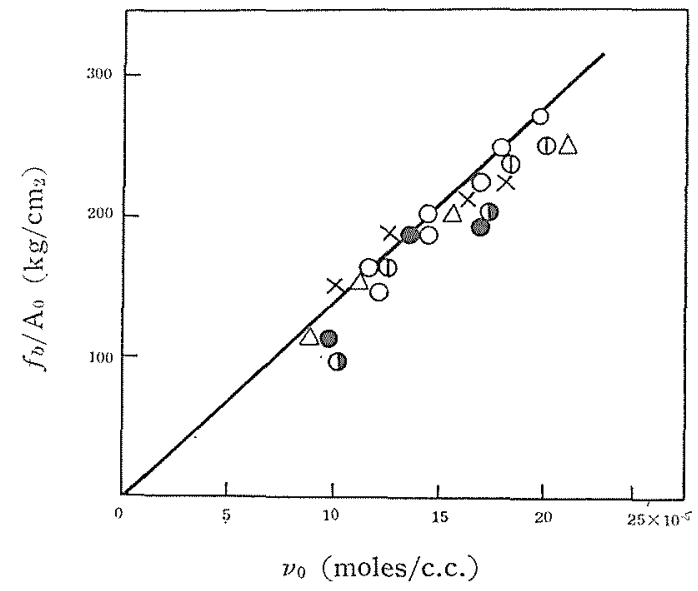

Fig. 10. Linear relationship between strength of vulcanized rubber and degree of cross-linking; the latter is measured by swelling methods (experiment in our laboratory ${ }^{92}$ ).

The good agreements with experiments are observed in all cases of vulcanized rubber except for that having exceedingly large degree of cross-linking. The calculated value of $\left(a / a^{*}\right) F_{0}$ is about $30 \mathrm{Kcal} /$ mole and if taking $100 \mathrm{Kcal} / \mathrm{mole}$ for $F_{0}$, i.e. carbon-to-carbon bond, $a^{*}$ is known to be a third of $a$. The discrepancy for highly cross-linked rubber may be ascribed to the defect in orientation or random distribution of applied stress as accounted by A.M. Buoche.,

\section{BRITTLE RUPTURE OF PLASTIC SUBSTANCE:}




\section{Junji FURUKAWA}

In foregoing paragraphs two types of rupture are discussed, i. e. plastic rupture which is applicable to plastic material and plastic rupture to cross-linked one. In this paragraph rupture of another type is described, which takes place for linear polymer by extremely rapid deformation. If the rate of deformation is so rapid that it is not followed sufficiently by relaxation, the polymer chain will break down. In this case the rupture may possibly occur at critical elongation $\lambda_{b}$ of polymer chain.

Equation (14) is transformed into equation (42) or equation (43), if deformation proceeds at constant rate $\dot{\alpha}$,

$$
\ln \lambda_{1}=\ln \alpha_{\mathrm{b}}-k_{0}{ }^{\prime}\left(\alpha_{\mathrm{h}}-1\right) / \alpha
$$

or

$$
\alpha_{1}=\left\{\lambda_{b} \exp \left(\alpha_{\mathrm{B}}-1\right) / \dot{\alpha} \tau\right\}
$$

equation (42) expresses the relation between the break length of specimen and the rate of deformation. Similarly the rupture time $t_{\mathrm{b}}$ is afforded by equation (44):

$$
t_{0}=\left(\lambda \mathrm{e} t_{\mathrm{w} / \mathrm{t}}-1\right) / \dot{\alpha} \text {. }
$$

If $t_{b}$ is smaller than the critical value concerning relaxation time or $1 / k_{0}{ }^{\prime}$, i.e.,

$$
t_{\mathrm{b}} / \tau<\left(1-1 / \lambda_{\mathrm{b}}, \mathrm{e}\right)
$$

it follows that:

$$
\begin{aligned}
& d \dot{\alpha} / d t_{\mathrm{b}},<0 \\
& \dot{\alpha} t_{\mathrm{b}} \cong \lambda_{\mathrm{b}}-1
\end{aligned}
$$

and

$$
\alpha_{b} \cong \lambda_{b} .
$$

The deformation rate should be smaller than relaxation rate constant $k_{0}{ }^{\prime}$ in plastic flow, otherwise it will undergo brittle rupture. Consequently the critical rate $\ddot{\alpha}_{\mathrm{c}}$ of deformation capable of inducing rupture is derived from equations (45) and (46):

$$
\begin{aligned}
\dot{\alpha}_{c} & =\left(\lambda_{b}-1\right)\left(1-1 / \lambda_{b} \mathrm{e}\right) \top \\
& \cong\left(\lambda_{\mathrm{b}}-1\right) \tau .
\end{aligned}
$$

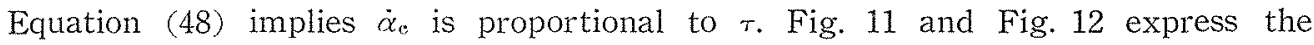
relation of $\alpha_{\mathrm{b}}$, and $t_{\mathrm{b}}$, to $\dot{\alpha}$.

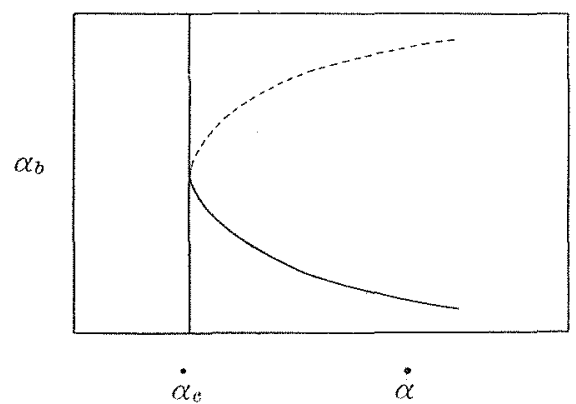

Fig. 11.

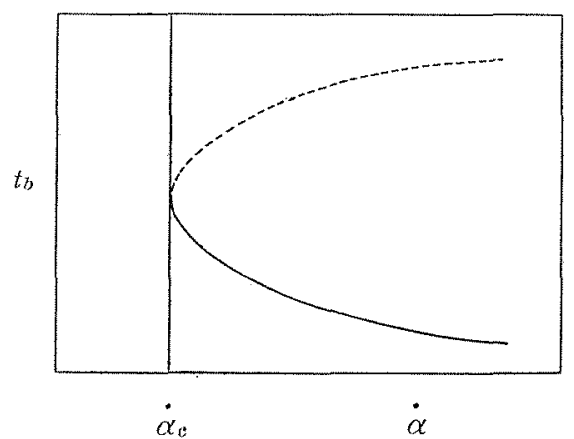

Fig. 12. 


\section{PROBLEMS IN ADHESIVES 7,6$)$}

The rupture in adhered speciemen takes place on the adhered interface or in the adhesive phase. Into both cases the plastic or elastic mechanism can be introduced. And the theoretical treatment is very similar to that mentioned above, though the former concerns surface phenomena unlike the latter. The bonded links on the interface $Z_{0}$ given by the following equation (49),

$$
\mathrm{Z}_{0}=\mathrm{M} /\left[1+\exp \left\{-\frac{\Delta F^{\prime}-\mathrm{N}_{0}(f / A) a^{*} / \mathrm{Z}_{0}}{R T}\right\}\right]
$$

Equation (49) is derived similary to equation (30), where number of functional group per unit surface is $\mathrm{M}$ and $\Delta F^{\prime}$ is bond dissociation energy for cross bond. And it can be concluded that the rupture may occur at the critical bonded links Ze,

$$
Z_{\mathrm{c}}=Z_{0} / \mathrm{e},
$$

and the bond strength is shown by equation (51):

$$
f_{c} / A=a^{*}\left(Z_{0} / \mathrm{M}\right)(1 / \mathrm{e}) R T \text {. }
$$

The time required for the separation of adhered specimen, $t_{\mathrm{b}}$, is expressed similarly by equation (52), which is similar to equation (37),

$$
t_{\mathrm{b}}=\left(Z_{0} / k_{0}{ }^{\prime}\right) \exp \left\{-\frac{f}{A} \frac{a^{*}}{2 Z_{0} k T}\right\}
$$

Fig. 13, which is calculated from the data of Hatfield et al., shows the above linear relationship between $\ln t_{\mathrm{b}}$ and $f$ for polyisobutene, where $Z_{0}$ is $4.4 \times 10^{11} \mathrm{~cm}^{-2}$ and the activation energy for rupture is $19.3 \mathrm{Kcal} / \mathrm{mole}$.

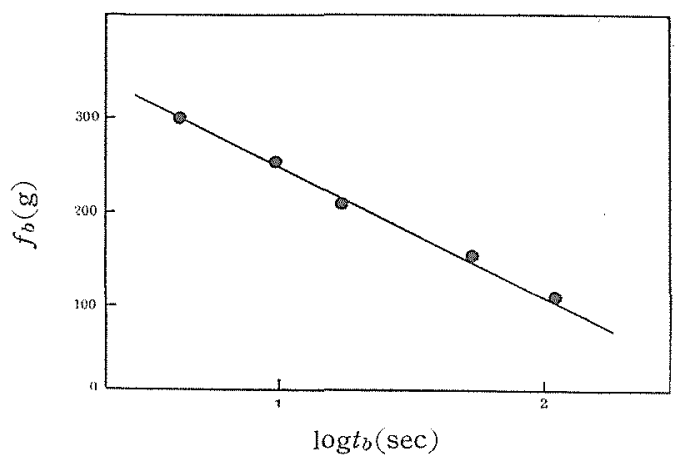

Fig. 13. The comparison between the equation (52) and Hatfield's data.

$$
\left(- \text {, equation (52); } 0 \text {, Hatfield's data }{ }^{10}\right)
$$

The rupture in adhesives layer is regarded quite similar to that mentioned above. However bonding strength is known to be small in the case of thick layer and this effect was explained in various ways, for examples by the defect in adhesive layer or the effect of long range force etc. The author found that the data was in good accordance with equation (53)

$$
\frac{1}{f_{\mathrm{b}}}=\frac{1}{f_{0}}+\frac{8 t_{\mathrm{b}}}{3 \eta R} D_{01}{ }^{2},
$$


as is shown in Fig. 13, where $f_{\mathrm{b}}$ is bonding strength after rupture time $t_{\mathrm{b}}$ for the bonding of two disks, whose diameter is $R$, and $D_{0}$ is the thickness of adhesives

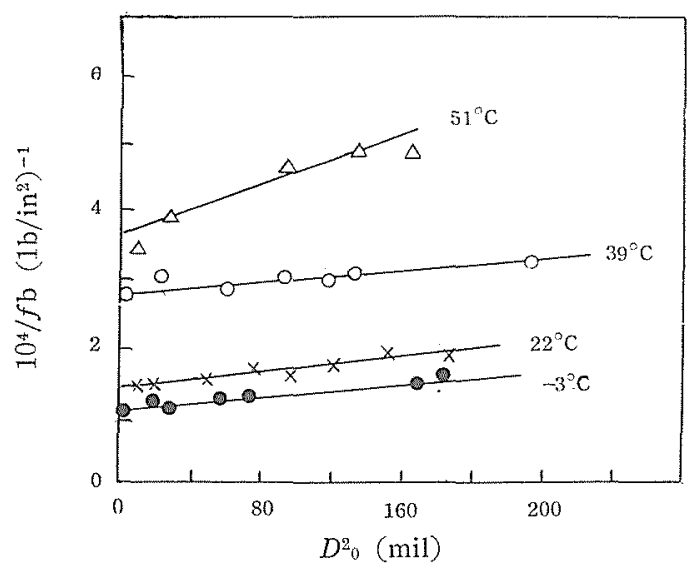

Fig. 14. Experiment in poly-vinylacetate-steel bonding ${ }^{11\rangle}$.

layer whose viscosity is $\eta$. Equation (53) involves the correction term as to the constant $f_{0}$ in the theoretical equation (54):

$$
\frac{1}{f_{\mathrm{b}}}=\frac{8 t_{\mathrm{b}}}{3 \eta R} D_{0}{ }^{2} \text {. }
$$

Equation (53) is very similar to the equation (55), which is well-known as the Maxwell equation:

$$
\frac{1}{f_{\mathrm{b}}}=\frac{1}{E \gamma_{\mathrm{b}}}+\frac{t_{\mathrm{b}}}{\eta \gamma_{\mathrm{b}}},
$$

where $E$ is elasticity and $\gamma_{\mathrm{b}}$ is deformation at break. Equation (55) suggests that adhesives layer consists of elastic layer and plastic layer. The elastic layer may be located near interface and therefore the thick adhesive tends to cause plastic flow and to have lower bonding strength. In equation (53) $f_{0}$ is the hypothetical strength where no plastic layer presents and it is proved to be the same as the strength of adhesives below its vitrification temperature. Equation (16) may be derived as follows:

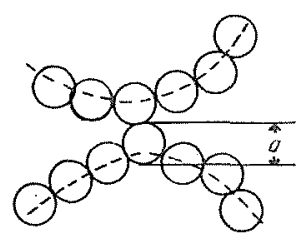

Initial state

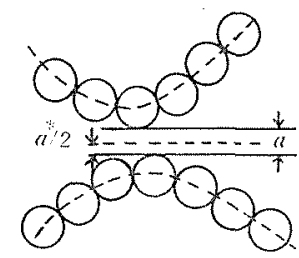

Transition state

Fig. 15.

In Fig. 15 the external force acts on each of linked segments, whose concentration is $\nu / N_{0}$ and whose cross-sectional area is $a^{2}$. Consequently the concentrated external force acting on one linked segment is that, 


$$
\begin{aligned}
& \mathrm{f} \times \frac{a^{2}}{\mathrm{~A}} \times \frac{1}{\nu / \mathrm{N}_{0}} \\
& \mathrm{~N}_{0}=1 / a^{3},
\end{aligned}
$$

where $N_{0}$ is number of segments having the volume of $a^{3} \mathrm{~cm}^{3}$ per one $\mathrm{cm}^{3}$ of the specimen. On the other hand, assuming that linking will break down, when linked segments are pulled apart by $a^{*} \mathrm{~cm}$ from their normal position, the work done on each linked segments is:

$$
\mathrm{W}=\frac{f a^{2}}{A \nu / \mathrm{N}_{0}} \times \frac{a^{*}}{2}=\frac{f}{A} \quad \frac{a^{*} / a}{2 \nu} .
$$

Hence the rate constant for bond breaking $k f^{\prime}$ is expressed by

$$
k_{\mathrm{f}}{ }^{\prime}=(k T / h) \exp \left(-\left(\varepsilon_{0}-\omega\right) / k T\right),
$$

if the work stated before may contribute to bond breaking. Where $\varepsilon_{0}$ is activation energy of bond breaking under no stress. Since $k_{0}{ }^{\prime}$ is

$$
k_{0}{ }^{\prime}=(k T / h) \exp \left(-\varepsilon_{0} / k T\right)
$$

it follows that

$$
k_{f}^{\prime}=k_{0}{ }^{\prime} \exp \left(-\frac{f}{A} \frac{a^{*} / a}{2 \nu k T}\right)
$$

\section{APPENDIX II.}

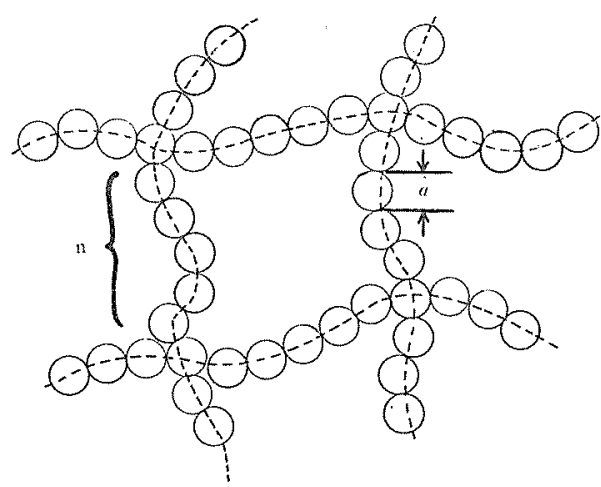

Fig. 16.

The statistical treatment shows that the mean distance of chain, which consists of $\mathrm{n}$ segment of length of $a \mathrm{~cm}$, is $\mathrm{n} a \mathrm{~cm}$. Therefore, the area occupied by one chain is $\mathrm{n} a^{2}$ and the number of chains per $1 \mathrm{~cm}^{2}$ is possibly expressed as:

$$
\nu_{0}{ }^{\prime}=1 / \mathrm{n} a^{2} \text {. }
$$

On the other hand the number of chains and that of segments per unit volume $\left(1 \mathrm{~cm}^{3}\right)$ of specimen are respectively,

$$
\begin{aligned}
& \nu_{0}=1 / \mathrm{n} \\
& \mathrm{N}_{0}=1 / a^{3}
\end{aligned}
$$

from equations (61) to (63) it follows that

$$
\nu_{0}{ }^{\prime}=\nu_{0} a \text {. }
$$




\section{Junji FURUKAWA}

\section{REFERENCES}

(1) A.V. Tobolsky, H. Eyring J. Chem. Phys., 11, 125 (1943).

(2) J. Furukawa, J. Polym. Sci., 15, 193 (1955)

(3) J. Furukawa, Collected paper read at the meeting held by the Japan Chemical Fibre Institute, Kyoto Univ., Vol. 13, 157 (1956).

(4) S. Glasstone, H. Eyring, "The Theory of Rate Processes" (1941).

(5) J. Furukawa and K. Muramatsu, unpublished.

(6) S. Midzushima et al. "High polymer", published by Kyoritzushuppansha (1949), p. 197.

(7) J. Furukawa, "Rheology for Chemical Engineer" published by Makishoten (1959) page

(8) J. Furukawa, K. Muramatsu, “Adhesives” publishd by Kobunshi-gakukai, (1959), p.84 203.

(9) J. Furukawa and S. Yamashita, J. Soc. Rubb. Ind. Japan, 30, 673 (1957).

(10) M.R. Hatfield and G.B. Rathmann, J. Phys. Chem., 60, 957 (1956).

(11) S.W. Lassoki, Jr. and G. Kraus, J. Polym. Sci., 18, 359 (1955).

(12) J. Furukawa, Jushikako (Resin Processing), 1956, Oct. 1. 